\title{
MIXING DUE TO ANGULAR MOMENTUM TRANSFER IN EVOLVING SUN- LIKE STARS
}

\author{
PIERRE DEMARQUE \\ Center for Solar and Space Research \\ Yale University \\ P.O. BOX 6666 \\ New Haven, CT 06511, USA
}

\begin{abstract}
The physical mechanisms that control the rotational history of stars like the Sun are reviewed, and the likely role of the associated rotational mixing is described. Results of stellar evolution calculations that include the effects of rotation are also described. The same theory, based on a single calibration for stars of different masses and metallicities, can successfully explain the observed $\mathrm{Li}$ and $\mathrm{Be}$ depletion in young open clusters and $\mathrm{Li}$ in field dwarf stars of the halo population, and the striking difference between metal-rich and metal- poor abundance patterns. There is evidence, both observational and theoretical, that angular momentum remains hidden in stellar interiors into advanced phases of evolution. Internal rotation also offers a natural explanation for the $\mathrm{CNO}$ isotopic anomalies observed among red giants. Finally, some of the questions raised by recent progress are considered.
\end{abstract}

\section{Introduction.}

The main factor that determine the rotational history of a sun-like star are its initial angular momentum $\mathbf{J}_{0}$, the nature of the angular momentum loss mechanism, and the nature of the internal angular transfer mechanisms.

\subsection{INITIAL ANGULAR MOMENTUM}

The initial total angular momentum $\mathrm{J}_{\mathrm{O}}$ of the star as it begins its hydrostatic evolution on the Hayashi line is given by the empirical Kraft (1970) curve. The Kraft curve is a plot of the minimum angular momentum of a star (since it is based on the assumption of rigid rotation in the interior) against mass. An important feature of the Kraft curve is the break which occurs just above one solar mass. Stars with masses lower than the break mass are observed to rotate slowly, while more massive stars are mostly rapid rotators. Indeed, the sun is well-known to rotate slowly. The original Kraft curve has recently been updated by Kawaler (1987), who showed that the angular momentum of the stars that define the Kraft curve corresponds to surface velocities approximately one third of break-up. It has long been recognized that the break in the curve coincides closely with the appearance of surface convection zones, and with the onset of magnetic activity for late-type stars along the main sequence (Wilson 1966; Demarque and Roeder 1966; Dicke 1970). Schatzman (1962) was the first to suggest that late-type stars (star with masses below the break in the Kraft curve) are slowed down by a torque exerted at their surface by the interaction of a stellar wind with 
the interstellar magnetic field. One must conclude that the break in the Kraft curve is not due to a change in the initial conditions of forming stars, but rather is the result of a difference in the rotational history.

\subsection{ANGULAR MOMENTUM LOSS}

The Schatzman mechanism (wind torque) is generally viewed as responsible for slowing down the outer layers of the star, and in turn for removing angular momentum from its interior. Mestel (1984) has devised a phenomenological model of the braking mechanism based on the magnetic field geometry, which was recently discussed and applied to stellar evolution by Kawaler (1987).

\subsection{ANGULAR MOMENTUM TRANSFER}

A number of dynamical instabilities are responsible for the angular momentum redistribution within the star as it evolves and changes its density distribution and moment of inertia. Most of these processes result in internal motions and chemical mixing which can modify the evolution and sometimes result in modifying initial surface abundances. The nature, characteristic time scales, and mixing efficiency of the main relevant processes will be discussed briefly in subsequent sections.

\section{Stellar Evolution with Rotation}

\subsection{BASIC APPROACH}

I will now describe calculations of the evolution of rotating sun-like stars. These calculations are carried out from the pre-main sequence fully convective Hayashi phase, and include the history of the star's internal angular momentum, and the associated mixing processes. Our approach is derived from that originally used by Endal and Sofia $(1976,1978,1981)$ in a series of ground breaking papers. Its implementation in the Yale stellar evolution code has been described in detail by Pinsonneault (1988) and Pinsonneault et al. (1989) (hereafter PKSD). The same technique, using the same calibration in all cases, has also been applied successfully to the study of young open clusters (Pinsonneault et al. 1990) (hereafter PKD) of the disk population, and to low metallicity halo stars (Deliyannis et al. 1989; Deliyannis and Pinsonneault 1990; Pinsonneault et al. 1991a,b). Its success in matching global rotational and composition constraints does not, of course, imply the correctness of all the simplifying assumptions made in the models. However it is an appealing feature of this approach that it has considerable predictive power, and that it yields consistent results for stars of a variety of masses, metallicities and evolutionary status. The main features of the Endal-Sofia (ES) approach are: (1) the structural distortions due to internal rotation are described in a quasi-spherical approximation, thus effectively reducing the problem to one dimension (see Kippenhahn and Thomas 1970; Law 1980, 1981); and (2) much care is devoted to the modeling of the mixing processes associated with the onset of rotationally induced instabilities. These instabilities, each of which has a different characteristic time-scale, are the factors that determine the evolution of the angular momentum inside stars. 


\subsection{SOME MODEL CHARACTERISTICS}

Let us now consider briefly some of the key features of the models. They are: the rotational breaking by stellar winds, the tre'tment of rotation in convective zones, the key role played by the transition layer at the interface between the base of the convection zone and the radiative interior, and finally, the nature and treatment of the rotationally induced instabilities in the radiative regions.

2.2.1. Rotational breaking by stellar winds. Schatzman (1962) introduced the idea of a stellar wind interacting with the interstellar magnetic field, and exerting a torque on the stellar surface. We have adopted the formalism due to Mestel (1984), as recently extended and implemented by Kawaler (1988), to describe the wind law. The wind law contains two free parameters: the wind index N, and the scaling factor $\mathrm{K}_{\mathrm{W}}$. $\mathrm{N}$ is a function of the magnetic field geometry. It was chosen to be 1.5 , which agrees best with the cluster data, and correspond to the empirically well-established Skumanich (1972) law for the oldest disk stars (which states that the surface rotational velocity of late-type main sequence stars decreases as the inverse square root of time). The other constant, $\mathrm{K}_{\mathrm{W}}$, can be set to match the Sun's surface rotation rate. Extensive sensitivity tests have been conducted in the contexts of both young disk stars and halo stars (Pinsonneault 1988).

\subsubsection{Rotating law in convection zones. We enforce rigid rotation in the convection zone on a} short timescale. We know that this assumption is not exactly correct, at least not in the outer parts of the solar convection zone, and perhaps not near its base. However, it must be closely satisfied in most of the convective zone. Furthermore, observations of the splitting of p-modes in the sun are consistent with nearly rigid rotation in most of the solar convection zone (see Demarque and Guenther 1988). That the assumption of rigid rotation represents a good approximation is confirmed by observations of pre-main sequence stars in young open clusters (Pinsonneault et al. 1990), and of the distribution of surface rotational velocities on the horizontal-branch of globular clusters (Pinsonneault et al. 1991a).

2.2.3. Importance of the transition layer. The existence of a transition layer between the radiative interior and the convective envelope is necessary for the efficient transport of angular momentum from the deep interior to the surface. Angular momentum is rapidly removed from the convection zone which rotates rigidly, and in the absence of a transition layer, too much angular momentum would remain trapped below the convection zone (Endal and Sofia, 1981). In the case of the sun, it would lead to an oblateness much in excess of the observational upper limit (Hill and Stebbins 1975). The strong shear that develops in this transition layer insures the steady and efficient transfer of angular momentum from the stellar core, and the associated mixing of chemical species throughout the evolving star.

2.2.4. Radiative interior. If the radiative core rotates, it is subject to meridional circulation. Since meridional circulation plays a role only when the rotation is sufficiently rapid, it is of little importance in the outer parts of late-type stars where rotational velocities are known to be low, due to the braking by the wind. Thus if late-type stars were rigid rotators, their surface rotation rates are such that meridional circulation would produce little or no mixing in them [see e.g. the discussion in Schwarzschild's (1958) textbook]. However, the inner parts of our models rotate more rapidly than their surface, and meridional circulation could be effective in mixing the interior, wherever it is not inhibited by gradients of mean molecular weight. The rotational models also predict rotation rate inside red giants sufficiently large for meridional circulation to operate 
effectively, even for the low-mass red giants found in globular clusters (Sweigart and Mengel 1979; Deliyannis et al. 1989; Pinsonneault et al. 1991a). During the pre-main sequence and main sequence phases, other rotationally induced mixing mechanisms dominate. Normal evolutionary processes tend to create differential rotation in the radiative inner core of stars as their core contracts and their envelope expands. It is known that internal velocity gradients can lead to shear induced turbulence and mixing. This is the idea behind the concept of "turbulent diffusion" which has been used in many studies of chemical mixing in stars (see e.g. Schatzman and Maeder 1981; Lebreton and Maeder 1986; Vauclair 1988). Because our approach differs from these in several important aspects, it is described in more detail in the next section.

\section{Physical Mechanisms for Angular Momentum Transfer and Mixing}

It is convenient to divide the instabilities that can result in angular momentum transfer and mixing according to the characteristic time-scale in which these instabilities operate. Following Endal and Sofia $(1978,1981)$ and PKSD, we shall divide them in two classes: dynamical instabilities that act on a very short time-scale, and therefore can be assumed to act instantaneously for the purpose of stellar evolution, and secular instabilities, which act on a longer time-scale, and which it is convenient to treat as a time-dependent diffusion process. A detailed discussion of the relevant mechanisms can be found in ES and PKSD. Only the most important mechanisms in the evolution of rotating sun-like stars are listed below.

\subsection{DYNAMICAL INSTABILITIES}

3.1.1. Convection. The onset of convection is determined, as in the non-rotating case, by the Schwarzschild criterion. Convective regions are chemically homogenized on a very short time-scale. The convective overtum timescale is of the order of weeks in solar-type stars. Convection zones tolerate little differential rotation, and therefore transport angular momentum outward very efficiently.

3.1.2. Dynamical shear. The dynamical shear is very effective in sun-like stars. It is the result of strong rotational gradients (Zahn 1974). It is stabilized by density gradients, and is responsible for preserving constant rotation on equipotential surfaces.

\subsection{SECULAR INSTABILITIES}

3.2.1. Meridional circulation. As mentioned earlier, meridional circulation, although treated in our models, plays very little role in sun-like stars during the pre-main sequence and main sequence phases. This is because rotation is relatively slow in most of the star. It is slowest, in particular, in the outer parts where the destruction of $\mathrm{Li}$ and Be take place. In the core, where rotation is more rapid, meridional circulation is inhibited by the presence of a gradient in mean molecular weight. In this regard, our work differs from that of Charbonneau and Michaud (1988) and Vauclair (1988) who assume that meridional circulation is the primary mechanism responsible for light element mixing and destruction. However, meridional circulation can play a role in stars more massive than the sun, which do not have deep convective envelopes (Charbonneau 1990), and on the giant branch where it can cause CNO mixing (Sweigart and Mengel 1979; Charbonneau 1990;

Pinsonneault et al. 1991a). 
3.2.2. The Goldreich-Schubert-Fricke (GSF) instability. This mechanism is primarily effective in the core, where radial velocity gradients are steep (Goldreich and Schubert 1967; Fricke 1968). It is also inhibited by mean molecular weight gradients, which are present in the core as a result of nuclear evolution. The GSF instability could be important in establishing the internal distribution profile of CNO processed isotopes near the main sequence that will eventually be mixed to the stellar surface, as the convection zone deepens on the giant branch.

3.2.3. The secular shear. The secular shear (Zahn 1974) arises because radiative diffusion tends to counter the stabilizing effect of density gradients. It is the dominant process in determining the radial gradient of rotation in the radiative envelope, below the surface convection zone. Its efficiency is determined by a critical Reynolds number whose value is uncertain in stars by an order of magnitude. Note however that the behaviour of the secular shear efficiency should be the same in all stars. Constraints on its efficiency can be applied from the observations of the observed rate of spindown in young open clusters (PKSD, PKD).

\subsection{TWO IMPORTANT POINTS ON ROTATIONAL INSTABILITIES}

Two important points must be noted regarding the action of various mixing mechanisms inside stars:

(1) Whereas the rate of meridional circulation depends on the rotation rate, shear instabilities and the GSF instability are functions of the radial gradient of the rotation rate. The resulting rotational history is affected in a sensitive way by these differences.

(2) The onset of the shear and GSF instabilities are subject to stability criteria which determine in each case a stability threshold. The mixing of angular momentum and chemical composition then takes place until stability is restored. During the course of stellar evolution, the effects of the instability are felt only when and where the instability occurs, and on the appropriate time-scale. This requires the detailed construction of complete evolutionary sequences which incorporate at each step and within each model the consequences of differential rotation on stellar structure, in an internally consistent way.

\subsection{CALCULATION OF DIFFUSION COEFFICIENTS}

The diffusion cofficient is the product of a length scale with a characteristic velocity. The length scale is taken to be the velocity scale height or the radius of the star, whichever is smaller. The velocity used for the diffusion coefficient is the sum of the circulation velocities generated by each secular instability. In the presence of a gradient in mean molecular weight, a stabilizing velocity is calculated and subtracted from the velocity sum. The same diffusion coefficient is used for the associated chemical mixing, multiplied by an efficiency factor $f_{c}$ (PKSD). The parameters that characterize each particular process are in some instances very uncertain, but once they have been chosen, in each case on the basis of some observational constraint, no other assumption needs to be made. The procedure thus ensures that a minimum of assumptions are needed. In addition, since the models are not tailored to explain just one particular set of observations by suitably adjusting free parameters, the procedure has considerable predictive power. The limits of application of the modeling assumptions can then be tested against a broad spectrum of observations. 


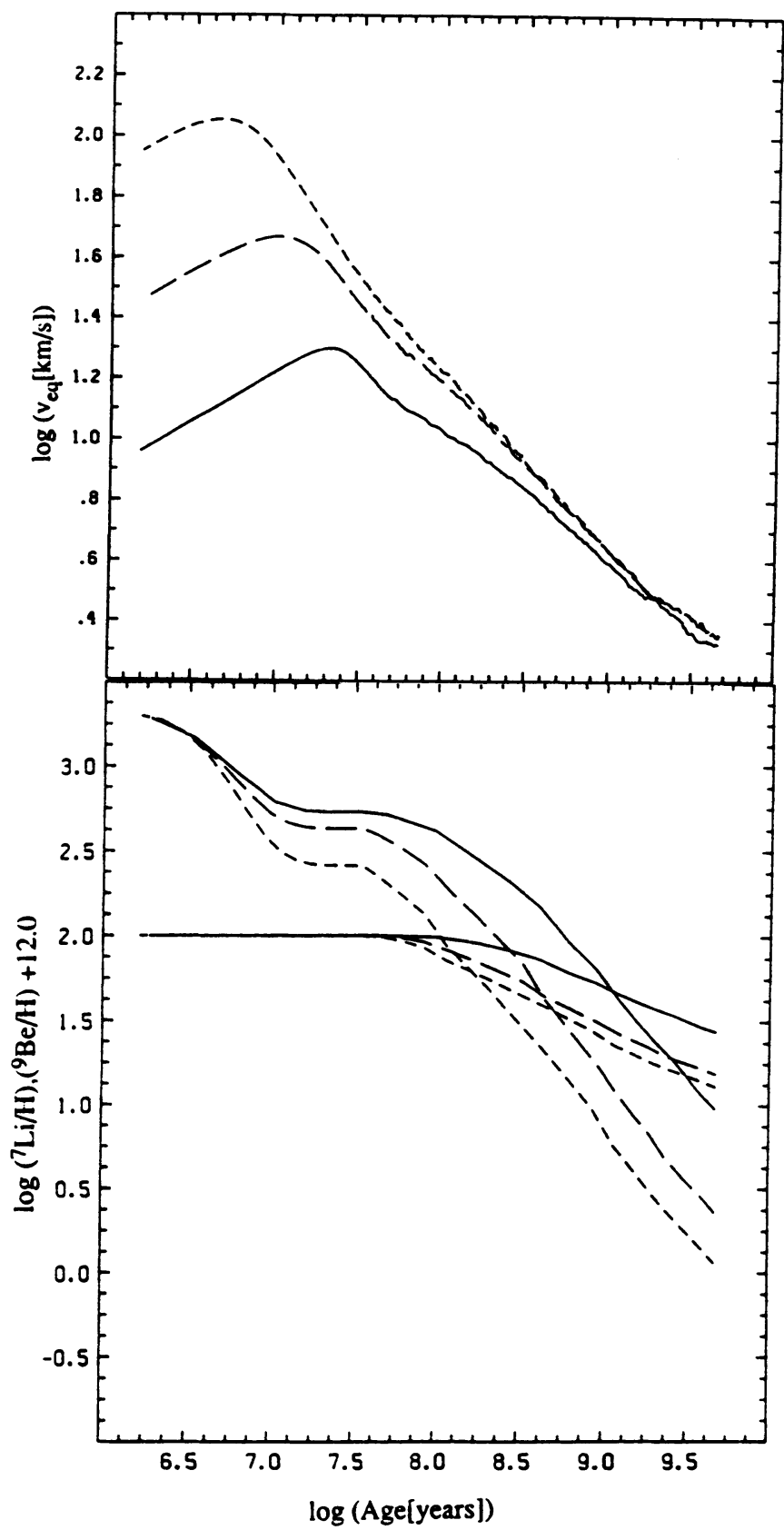

Figure 1. The upper panel shows the variation with time of the equatorial rotational velocity of a model with the mass of the sun, for three values of the initial angular momentum: the "best" solar model value, and about ten times and one half the solar value. The lower panels illustrates the corresponding variation of the $\mathrm{Li}$ and $\mathrm{Be}$ surface abundances. 


\section{Comparison with Observation}

\subsection{THE SOLAR CALIBRATION AND OBSERVATIONS OF YOUNG OPEN CLUSTERS}

4.1.1. The solar calibration. The method described above has been applied to the study of the evolution of the rotating sun (PKSD). As explained above, a solar-stellar approach was used in constraining the five adjustable parameters in the calibration. The first two parameters for the stellar wind, $\mathbf{N}$ and $\mathrm{K}_{\mathrm{W}}$ have been described in Section 2.2.1. In addition, the total initial angular momentum for the sun is set so as to place correctly the solar $\mathrm{Li}$ abundance relative to solar analogs, starting from the Kraft curve; the parameter $f_{c}$, which is a measure of the efficiency of mixing compared to angular momentum transfer, is adjusted so as to match the estimated solar Li depletion; and finally the critical Reynolds number, to be used in the secular shear calculation, is chosen so as to reproduce best the spin-down of young cluster stars. Changes in any one of the adjustable parameters must be accompanied by suitable changes in the other parameters to preserve the solar calibration. A fiducial "best" solar model was adopted. We note that the "best" solar model parameters also yield the correct solar Be abundance, which cannot easily be done in models depleted by meridional circulation. Models for stars with different masses, chemical compositions and initial angular momenta were then constructed using these parameters.

\subsubsection{Young open clusters. Young open clusters, for which observations of ages and Li} abundances have become available in the last few years, provide an excellent series of snapshots of the past evolution of the sun and sun-like stars. The validity of the theoretical procedure is best illustrated by a simultaneous comparison of observations of surface rotational velocities and light element abundances of stars of different temperatures within each star cluster. We must first note that standard (non-rotating) stellar evolution calculations are unable to account for the observed depletion in $\mathrm{Li}$ and $\mathrm{Be}$ in star clusters and in the sun. The early stages are characterized by the competition between the contraction of the stellar radius, which spins up the star, and the torque exerted at the surface by the stellar wind. This is illustrated in the upper panel of Fig.1, which shows the resulting changes in surface velocities as a function of time for three choices of $\mathrm{J}_{\mathbf{0}}$. Because stars with the highest surface rotation rates are subject to the largest wind torques, all surface velocities asymptotically decrease to a value which is independent of the initial conditions, and depends only on form of the wind law. For $\mathrm{N}=1.5$, the wind law simulates the well-known empirical Skumanich law, as illustrated in the upper panel of Fig.1.

As the evolution proceeds near the main sequence, the convection zone becomes more shallow. While the convection zone is spun down, angular momentum is also progressively removed from the radiative core by the action of the dynamical shear at the interface between the core and the envelope. During this phase, $\mathrm{Li}$ and $\mathrm{Be}$ also undergo depletion, as described in the lower panel of Fig.1. Note that the initially most rapid rotators are the ones that lose most angular momentum, and as a consequence, suffer the largest depletion in $\mathrm{Li}$ and $\mathrm{Be}$. In a stellar system of a given age, the $\mathrm{Li}$ abundance at a certain $\mathrm{T}_{\text {eff }}$ is thus a measure of the initial angular momentum $\mathrm{J}_{0}$, and the dispersion in $\mathrm{Li}$ abundance is a measure of the dispersion in $\mathrm{J}_{0}$. This is consistent with observations of $\mathrm{Li}$ abundances in star clusters of different ages (see detailed comparisons in $\mathrm{PKD}$ ). Theory predicts that the $\mathrm{Li}$ dispersion increases toward low masses, and that this effect is more pronounced in the younger clusters, a conclusion which agrees with observation (Balachandran 1988; PKD). For very young stars, however, this picture is an oversimplification. In some cases, the most rapid rotators are also the richest in $\mathrm{Li}$ (Rebolo 1990). Possible interpretations for the 
observations are discussed briefly in Section 5.

\subsection{SUBGIANTS}

Observations of surface rotation periods of subgiant with masses near that of the sun offer a direct test of the radial rotation curve of sun-like stars near the main sequence. As the surface convection zone deepens and increases in mass, it acquires by dredge-up the angular momentum of the deeper layers. Since convection enforces rigid rotation on a short time-scale, the angular momentum is redistributed and the surface rotation is higher than it would be in the absence of rapid internal rotation. Fig.2 illustrates this effect for the PKSD solar model. It shows that the observations of Noyes et al (1984) are compatible with the prediction of the PKSD model, and disagree with the prediction of a rigidly rotating solar model. A discussion of this point in the context of solar seismology is given by Demarque and Guenther (1988).

\subsection{HALO DWARF STARS}

Field halo dwarfs and main sequence stars in globular clusters share with the sun and sun-like disk stars the presence of a surface convection zone, which controls their rotational history. On the other hand, because of their low metallicities, halo dwarfs have a different mass-luminosity law and for a given mass, much less massive convection zones. These two factors affect their rotational histories in a dramatic way (Pinsonneault et al. 1991a). Calculations performed for the appropriate parameters of low-metallicity stars, and using the same rotational parameters as in the solar calibration, yield relatively more rapidly rotating cores in halo than in solar-metallicity stars. The amount of angular momentum retained, although insufficient to affect the shape of the evolutionary track in the HR-diagram or the main sequence lifetime, is however sufficient to explain the observations of rotational velocities of horizontal-branch stars in globular clusters (Pinsonneault et al. 1991a). In addition, it is compatible with the requirements for the mixing of CNO processed elements to the surface of red giant stars. This important result is currently being explored in greater detail. Finally, it is interesting to note that for extreme halo dwarfs, the characteristic Spite plateau in the $\mathrm{Li}$ abundance as a function of $\mathrm{T}_{\text {eff }}$ is preserved even in the presence of rotation, although its level is lowered compared to non-rotating stars, by a factor which could be as high as nearly one order of magnitude (Pinsonneault et al. 1991b). This result is important in constraining the primordial $\mathrm{Li}$ abundance. In addition, the bluest extent and the flatness of the Spite Li plateau are both a function of age. The $\mathrm{Be}$ abundance in halo stars provides another important constraint to cosmology and big bang nucleosynthesis (Deliyannis and Pinsonneault 1990).

\section{Some Successes, Uncertainties and Future Challenges.}

\subsection{SUCCESSES OF THE ROTATION THEORY}

We have described an approach to calculate the evolution of the angular momentum distribution in stars which yields remarkable agreement with observations of light element abundances (both $\mathrm{Li}$ and $\mathrm{Be}$ ) and surface rotation velocities in young star clusters. This approach also predicts a radial rotation profile in sun-like stars which is consistent with the observational evidence for dredge-up of angular momentum as the surface convection zone deepens in the subgiant phase of evolution. The prediction for the rotational properties of metal-poor halo dwarfs is also consistent with 


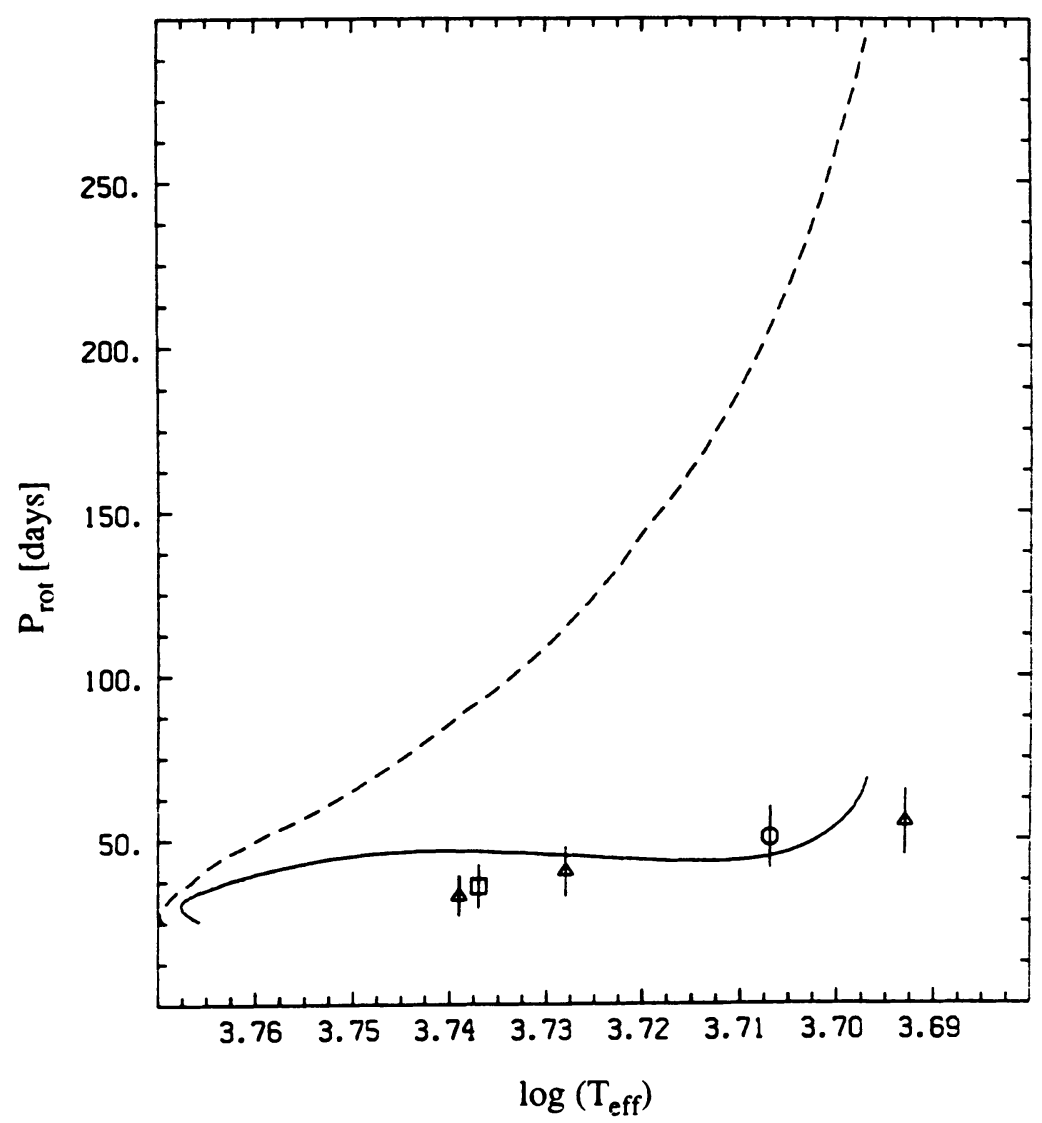

Figure 2. Subgiant evolution of two rotating models. The solid line shows the evolution of the PKSD model. The dashed line stands for a rigidly rotating model. The points are from the observations of Noyes et al. (1984). 
observations of rotational velocities of horizontal-branch stars, and with the conclusion that CNO isotopic anomalies in red giants are the result of rotational mixing. Models near the turnoff also permit to draw some conclusions concerning the timing and the nature of the mixing of CNO processed elements to the surface. The differences in structure and intemal rotation profile between solar-metallicity and low-metallicity stars offer a natural explanation of their observed differences in CNO processing and mixing. In solar metallicity stars, internal mixing of CNO processed elements during main sequence evolution, followed by dredge-up at the base of the giant branch, yields the ratios of $\mathrm{C}$ and $\mathrm{O}$ isotopes observed in red giant stars. By contrast, models for metal-poor stars suggest that these stars suffer, in addition to the giant-branch dredge-up of CNO elements, further CNO processing during giant evolution. Such differences are observed in metal-rich and metalpoor star clusters (e.g. M67 and M92).

\subsection{SOME UNCERTAINTIES AND CHALLENGES}

5.2.1. Efficiency of mixing. In the discussion of angular momentum transfer, a major uncertainty remains the efficiency factor $f_{c}$ for material mixing associated with angular momentum transfer. For simplicity, this factor was taken as a constant (an average over time and position) during evolution for each class of physical processes. This assumption cannot be strictly correct, and is probably very wrong in some situations. One also notes that in order to match the solar Li abundance, $\mathrm{f}_{\mathrm{c}}$ must be significantly less than unity (we use 0.046 in our "best" solar calibration). This finding is in accord with other discussions of turbulent mixing and angular momentum transfer by Schatzman (1987) and by Tassoul and Tassoul (1989). Some might then take the extreme view that the low value of $f_{c}$ required to match the observations is an indications that mixing and angular momentum transfer are practically uncoupled, and are due to unrelated processes. It seems more likely that a low $\mathrm{f}_{\mathrm{c}}$ is simply a hint that magnetic fields, and/or waves, may transport much of the angular momentum out of the star (Fox and Bernstein 1987; Spruit 1987; Zahn 1987). The encouraging agreement with observation, achieved over a range of masses, ages and chemical compositions, is a compelling indication that there exists indeed a relation between the observed mixing and the history of angular momentum transfer in stars, even though the precise form of this relation is more complex than current modeling assumes. Understanding this relation remains one of the major tasks ahead.

5.2.2. The wind law. In all cases presented here, a unique wind law was adopted. The two free parameters $\mathrm{N}$ and $\mathrm{K}_{\mathrm{W}}$ were calibrated on the observations of the surface rotation of stars in clusters and on the sun, respectively. In addition, $\mathrm{N}=1.5$ yields the Skumanich law derived for sun-like dwarfs of different ages. For these reasons, and for the sake of simplicity, the two free parameters were kept fixed. However, one must remain conscious of the assumptions of the models. In particular, different magnetic field configurations are likely to dominate in the very early pre-main sequence phases, which are characterized by strong magnetic activity and high rotation rates (e.g. T Tauri stars). Such young stars are also known to be surrounded by accretion disks (Strom et al. 1989), which must modify both the surface rotation and the $\mathrm{Li}$ abundance in ways that are not yet understood. Indeed some observations of very young stars do confirm the suspicion that different physics must apply [e.g. the observations of strong Li coupled with rapid rotation reported at this Symposium by Rebolo (1990), which cannot be explained by the present models].

5.2.3. Interaction between rotation and microscopic diffusion. Deliyannis et al. (1990) have recently published preliminary results of microscopic diffusion calculation of helium in stellar 
envelopes near the turn-off of globular clusters for non-rotating stars. Other research, which in addition includes a treatment of helium diffusion in the whole stellar interior, has also been presented by Proffitt et al. (1990). We are currently exploring (with the help of graduate student B. Chaboyer) the sensitivity of theoretical isochrones near the main sequence turnoff to the adopted parameters. We note that in the presence of rotation, different helium diffusion patterns may be expected. These cannot be easily approximated without the help of realistic models which include a detailed treatment of the interaction between the mixing induced by the rotation and microscopic diffusion. Although helium cannot easily be observed in sun-like stars, Li diffusion can be used as a tracer of the efficiency of helium diffusion. This problem is particularly significant in view of the possible importance of diffusion for determining the absolute ages of globular star clusters (Stringfellow et al. 1983).

This work was supported in part by NASA grants NAGW-777 and NAGW-778 to Yale University.

\section{References}

Balachandran, S. (1988) "Lithium Depletion and Rotation in Main Sequence stars", Ph.D.

Dissertation, University of Texas.

Charbonneau, P. (1990) "Modélisation Numérique des Processus de Transport dans les

Enveloppes Stellaires", Thèse de Doctorat, Université de Montréal.

Charbonneau, P. and Michaud, G. (1988) "Meridional Circulation and the Li Abundance Gap in F

Stars", Astrophys. J. 334, 746-760.

Deliyannis, C.P. (1990), Ph.D. Dissertation, Yale University.

Deliyannis, C.P., Demarque, P. and Kawaler, S.D. (1990) "Lithium in Halo Stars from Standard

Stellar Evolution", Astrophys. J. Suppl. 73, 21-65.

Deliyannis, C.P., Demarque, P. and Pinsonneault, M.H. (1990) "The Ages of Globular Cluster

Stars: Effects of Rotation on Pre-main Sequence, Main Sequence, and Turnoff Evolution", Astrophys. J. Letters 347, L73-L76.

Deliyannis, C.P. and Pinsonneault, M.H. (1990) "Beryllium in the Galactic Halo: Surface Abundances from Standard, Diffusive and Rotational Stellar Evolution, and Implications", Astrophys. J. Letters 365, L67-L70.

Demarque, P. and Guenther, D.B. (1988) "Is the Sun Really a Rigid Rotator?", in V. Domingo and E.J. Rolfe (eds.), Proc. Symp. Seismology of the Sun and Sun-like Stars, Tenerife, Spain, ESA-286, pp. 99-102.

Demarque, P. and Roeder, R.C. (1967) "Hydrogen Convection Zones and Stellar Rotation", Astrophys. J. 147, 1188-1191.

Dicke, R.H. (1970) "Internal Rotation of the Sun", Ann. Rev. Astr. Astrophys. 8, 297-328.

Endal, A.S. and Sofia, S. (1976) "The Evolution of Rotating Stars.I.", Astrophys. J. 210, 184-198.

Endal, A.S. and Sofia, S. (1978) "The Evolution of Rotating Stars.II.", Astrophys. J. 220, 279-290.

Endal, A.S. and Sofia, S. (1981) "Rotation in Solar-type stars.I. Evolutionary Models for the Spin-down of the Sun", Astrophys. J. 243, 625-640.

Fox, P.A. and Bernstein, I.B. (1987) "The Generation of Magnetic Fields in the Sun", in B.R.

Durney and S. Sofia (eds.), The Internal Solar Angular Velocity, Reidel, Dordrecht, pp. 213-220.

Fricke, K. (1968) "Instabilität stationärer Rotation in Sternen", Zeit. Astrophys. 68, 317-344.

Goldreich, P. and Schubert, G. (1967) "Differential Rotation in Stars", Astrophys. J. 150,

571-587.

Hill, H.A. and Stebbins, R.T. 1975 "The Intrinsic Visual Oblateness of the Sun", Astrophys. J. 
200, 471-483.

Kawaler, S.D. (1987) "Angular Momentum in Stars: the Kraft Curve Revisited", Pub. Astr. Soc. Pacific 99, 1322-1328.

Kawaler, S.D. (1988) "Angular Momentum Loss in Low-mass Stars", Astrophys. J. 333, 236-247.

Kippenhahn, R. and Thomas, H.-C. (1970) "A Simple Method for the Solution of the Stellar Structure Equations including Rotation and Tidal Forces", in A. Slettebak (ed.), Stellar Rotation, Reidel, Dordrecht, pp. 20-29.

Kraft, R.P. (1972) "Stellar Rotation", in G.H. Herbig (ed.), Spectroscopic Astrophysics, University of California Press, Berkeley, pp. 385-422.

Law, W.-Y. (1980), Ph.D. dissertation, Yale University.

Law, W.-Y. (1981) "The Effect of Differential Rotation on the Internal Structure of Low-mass Stars" Astr. Astrophys. 102, 178-190.

Lebreton, Y. and Maeder, A. (1987) "Stellar Evolution with Turbulent Diffusion Mixing", Astr. Astrophys. 175, 99-112.

Mestel, L. (1984) "Angular Momentum Loss during Pre-main Sequence Contraction", S.L. Baliunas and L. Hartmann (eds.), in Cool Stars, Stellar Systems and the Sun, Springer-Verlag, Berlin, pp. 49-59.

Michaud, G. (1986) "The Lithium Abundance Gap in the Hyades F Stars: the Signature of Diffusion", Astrophys. J. 302, 650-655.

Michaud, G., Fontaine, G. and Beaudet, G. (1984) "The Lithium Abundance: Constraints on Stellar Evolution", Astrophys. J. 282, 206-213.

Noyes, R.W., Hartmann, L.W., Baliunas, S.L., Duncan, D.K. and Vaughan,A.H. 1984

"Rotation, Convection, and Magnetic Activity in lower Main Sequence Stars", Astrophys. J. 279, 763-777.

Pinsonneault, M.H. (1988) "Evolutionary Models of the Rotating Sun", Ph.D. Dissertation, Yale University.

Pinsonneault, M.H., Deliyannis, C.P., Demarque, P. (1991a) "Evolutionary Models of Halo Stars with Rotation:.I. Evidence for Differential Rotation with Depth in Stars", Astrophys. J., in press.

Pinsonneault, M.H., Deliyannis, C.P., Demarque, P. (1991b) "Evolutionary Models of Halo Stars with Rotation:.II. Effects of Metallicity on Lithium Depletion, and Possible Implications for the Primordial Lithium Abundance", Astrophys. J., in preparation.

Pinsonneault, M.H., Kawaler, S.D. and Demarque, P. (1990) "Rotation of Low-mass Stars: A New Probe of Stellar Evolution", Astrophys. J. Suppl. 74, 501-550.

Pinsonneault, M.H., Kawaler, S.D., Sofia, S. and Demarque, P. (1989) "Evolutionary Models of the Rotating Sun", Astrophys. J. 338, 424-452.

Proffitt, C.R., Michaud, G. and Richer, J. (1990) "Diffusion in Metal-poor Low-mass Stars", in G. Wallerstein (ed.), ASPCS Vol. 9, Brigham Young University Press, Provo, Utah, pp. 351-353.

Rebolo, R. 1990, these Proceedings.

Schatzman, E. (1962) "A Theory of the Role of Magnetic Activity during Star Formation", Ann. d'Astrophys. 25, 18-29.

Schatzman, E. (1987) "Solar Rotation and Age", in B.R. Durney and S. Sofia (eds.), The Internal Solar Angular Velocity, Reidel, Dordrecht, pp. 159-172.

Schatzman, E. and Maeder, A. (1981) "Solar Neutrinos and Turbulent Diffusion", Nature 290, 683-686.

Schwarzschild, M. (1958) Structure and Evolution of the Stars, Princeton University Press, Princeton, New Jersey.

Skumanich, A. (1972) "Timescales for Call Emission Decay, Rotational Braking and Lithium Depletion", Astrophys. J. 171, 565-567.

Spruit, H.C. (1987) "Angular Momentum Transport in the Radiative Interior of the Sun", in B.R. Durney and S. Sofia (eds.), The Internal Solar Angular Velocity, Reidel, Dordrecht, pp. 185-200. Stringfellow, G.S., Bodenheimer, P., Noerdlinger, P.D. and Arigo, R.J. (1983) "Evolutionary Effects of Helium Diffusion in Population II Stars", Astrophys. J. 264, 228-236.

Strom, K.M., Wilkin, F.P., Strom, S.E. and Seaman, S.D. (1989) "Lithium Abundances Among 
Solar-type Pre-main Sequence Stars", Astr. J. 98, 1444-1450.

Sweigart, A.V. and Mengel, J.G. (1979) "Meridional Circulation and CNO Anomalies in Red Giant Stars", Astrophys. J. 229, 624-641.

Tassoul, J.-L. and Tassoul, M. (1989) "The Internal Rotation of the Sun", Astr. Astrophys. 213, 397-401.

Vauclair, S. (1988) "Lithium Destruction in Stellar Outer Layers", Astrophys. J. 335, 971-975.

Wilson, O.C. 1966 "Stellar Convection Zones, Chromospheres, and Rotation", Astrophys. J. 144, 695-708.

Zahn, J.-P. (1974) "Rotational Instabilities and Stellar Evolution", in P. Ledoux, A. Noels and A.W. Rodgers (eds.), IAU Symposium No. 59, Stellar Instability and Evolution, Reidel, Dordrecht, pp.185-194.

Zahn, J.-P. (1987) "Turbulent Transport in the Radiative Zone of a Rotating Star", in B.R. Durney and S. Sofia (eds.), The Internal Solar Angular Velocity, Reidel, Dordrecht, pp. 201-212. 